\title{
Measurement of N-V Centers in Nanodiamond Particles using Advanced (S)TEM
}

Shery L. Y. Chang ${ }^{1}$, Katia March ${ }^{1}$, Christian Dwyer ${ }^{2}$

${ }^{1 .}$ LeRoy Eyring Center for Solid State Science, Arizona State University, Tempe, AZ 85287, United States.

2. Department of Physics, Arizona State University, Tempe, AZ 85287, United States.

Nitrogen-vacancy centers $(\mathrm{N}-\mathrm{V})$ in diamond have been studied extensively for their interesting photoluminescence properties. Negatively-charged $\mathrm{N}-\mathrm{V}$ centers $\left(\mathrm{N}-\mathrm{V}^{-}\right)$can emit visible light that is readily detectable at the room temperature. Spectroscopy techniques including Raman and particularly electron paramagnetic resonance (EPR) have been used to identify different types of defect centers in diamond [1]. Locating the optically active $\mathrm{N}-\mathrm{V}$ centers $\left(\mathrm{N}-\mathrm{V}^{-}\right)$, has becoming routine using optical fluorescence microscopy. Combined with AFM, the locations of the $\mathrm{N}_{-} \mathrm{V}^{-}$as well as the particle size can now be correlated [2]. However, with the continuing effort of developing N-V containing nanoparticles with smaller size $(<10 \mathrm{~nm})$, the understanding of how the host structure and the surface chemistry and structure becomes critical.

Here we investigate these effects by using high-resolution imaging and electron energy loss spectroscopy in the (S)TEM. We examined diamond nanoparticles synthesized using the detonation (DND) and high pressure high temperature (HPHT) processes. The surfaces of former particles were treated in order to improve the fluorescence property. The latter particles were treated with different concentrations of nitrogen doping as well as high energy electron irradiation and annealing.

High-resolution imaging was carried out using a monochromated, aberration-corrected TEM (Titan, FEI Company) operated at $80 \mathrm{kV}$. A monochromated, aberration corrected STEM (Nion UltraSTEM ${ }^{\mathrm{TM}}$ ) operated at $60 \mathrm{kV}$ was used for electron energy loss spectroscopy. The low electron beam energies minimize atomic structural damage.

The as-synthesized and surface modified detonation nanodiamond particles show different surface structures from HPHT nanodiamond. The DNDs exhibit fullerene-like surface structures, which is the result of surface relaxation due to the small particle size. This is also confirmed by the density functional tight-binding calculations [3]. The HPHT particles, on the other hand, show a range of size distribution and shapes, as shown in Fig. 1.

Fig. 2(a) shows experimental carbon K-edge spectra from DND, HPHT and reference bulk diamond and graphite. The near edge structure of HPHT particles (Fig. 2(b)), shows a pre-edge peak (282.5eV) that we have identified as associated with the point defects, including isolated vacancies and N-V centers. Using the theoretically calculated reference spectrum, we can quantify the defect concentration to be about $0.02 \%$. Moreover, we have carried out the low-loss EELS to investigate the electronic transitions arising from the $\mathrm{N}-\mathrm{V}$ centers.

We show the EELS can be used to identify the presence of $\mathrm{N}-\mathrm{V}$ centers in nanodiamond in individual nanodiamond particles. With the combination of high-resolution TEM imaging, the effect of surface modification and dopant treatment can be correlated. 


\section{References:}

[1] S Felton et al, Phys. Rev. B 77 (2008) p. 081201.

[2] C Bradac et al, Nature Nanotechnology 5 (2010) p. 345.

[3] S. L Y. Chang et al, Nanoscale Horizons (2018) 10.1039/C7NH00125H.
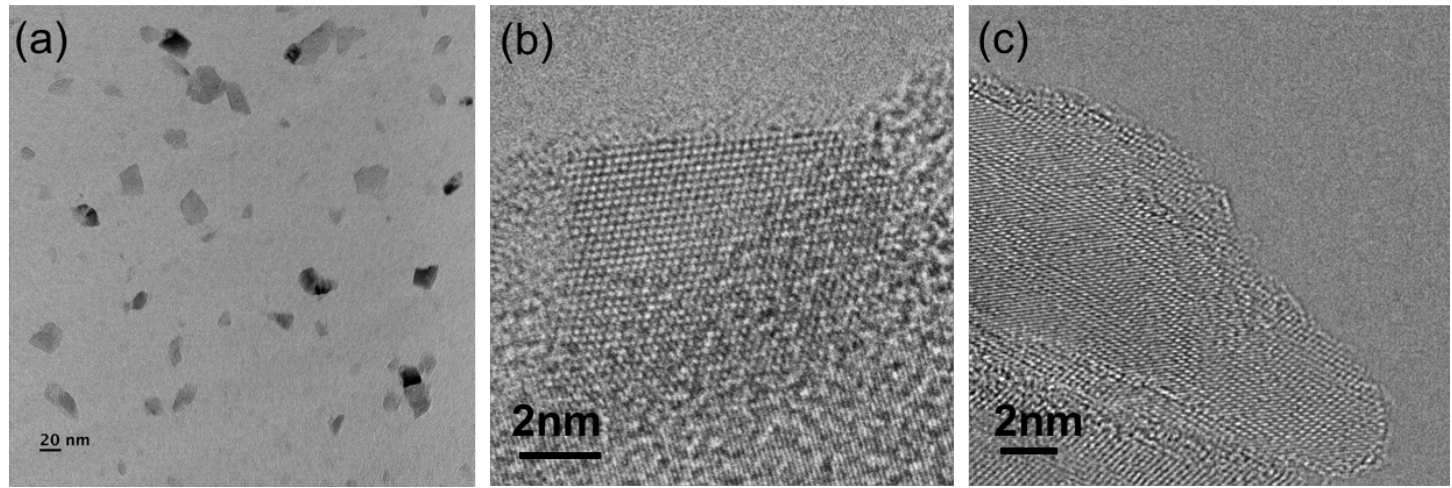

Figure 1. HRTEM images of nanodiamond particles synthesized through HPHT process, taken using a monochromated, aberration-corrected Titan operating at $80 \mathrm{kV}$. (a) Distribution of HPHT nanodiamond particles, (b) $8 \mathrm{~nm}$ nanoparticle showing primarily $\{111\}$ facets, and (c) $20 \mathrm{~nm}$ nanodiamond particle showing elongated shape with fragmented graphitic surfaces.
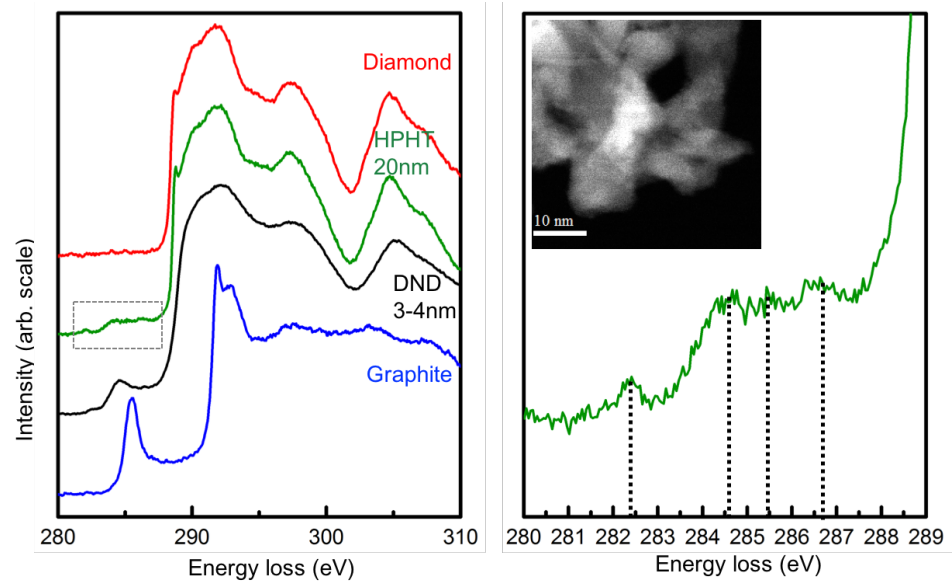

Figure 2. Monochromated carbon K-edge EEL spectra from HPHT and DND particles, showing pre-edge peaks indicative of vacancies and surface relaxation. 